\title{
Conjugated linoleic acid prompts bone formation in ovariectomized osteoporotic rats and weakens osteoclast formation after treatment with ultraviolet B
}

\author{
Zhongshu Shan ${ }^{1,2}$, Yanyan Zhao ${ }^{2}$, Zhixue Qiu ${ }^{2}$, Suonan Angxiu ${ }^{2}$, Yong Gu ${ }^{1}$, Junming Luo ${ }^{3}$, Hongtao Bi ${ }^{4}$, \\ Wei Luo ${ }^{5}$, Rui Xiong ${ }^{6}$, Siqing $\mathrm{Ma}^{7}$, Zhao $\mathrm{He}^{2}$, Liang Chen ${ }^{1}$ \\ ${ }^{1}$ Department of Orthopedic Surgery, the 1st Affiliated Hospital of Soochow University, Suzhou, China; ${ }^{2}$ Department of Orthopedic Surgery, People's \\ Hospital of Qinghai Province, Xining, China; ${ }^{3}$ Department of Pathology, People's Hospital of Qinghai Province, Xining, China; ${ }^{4}$ Qinghai Provincial \\ Key Laboratory of Tibetan Medicine Pharmacology and Safety Evaluation, Northwest Institute of Plateau Biology, Chinese Academy of Sciences; \\ Xining, China; 'Department of Endocrinology, People's Hospital of Qinghai Province, Xining, China; ${ }^{6}$ Nutrition Department, People's Hospital of \\ Qinghai Province, Xining, China; ${ }^{7}$ Department of Critical Care Medicine, People’s Hospital of Qinghai Province, Xining, China \\ Contributions: (I) Conception and design: H Bi, S Ma, W Luo, R Xiong; (II) Administrative support: None; (III) Provision of study materials or \\ patients: None; (IV) Collection and assembly of data: None; (V) Data analysis and interpretation: None; (VI) Manuscript writing: All authors; (VII) \\ Final approval of manuscript: All authors. \\ Correspondence to: Doctor Liang Chen. Department of Orthopedic Surgery, The First Affiliated Hospital of Soochow University, 188 Shizi St. Suzhou \\ 215006, China. Email: chenliangspine@163.com.
}

Background: Ultraviolet B (UVB) has been reported to prevent bone loss by promoting the synthesis of vitamin D. However, UVB can also enhance osteoclastic differentiation, inhibit osteogenic differentiation, and cause oxidative damage. The present study aimed to analyze the osteoprotective effects of UVB and conjugated linoleic acid (CLA) in rats with ovariectomy-induced osteoporosis, and to determine the interactions between UVB and CLA and their effects on bone mesenchymal stem cells (BMSCs) and bone marrow mononuclear cells (BMMCs).

Methods: In vitro, the distance of UVB irradiation and the dose of CLA were selected by immunofluorescence assays and Cytotoxicity assay. BMSCs and BMMCs were detected by immunohistochemical and immunofluorescence assays. In vivo, three-month-old female Sprague-Dawley rats that had undergone ovariectomy were treated with UVB and CLA. After 8 weeks of therapy, the femurs of the rats were examined by micro-computed tomography (CT) and immunohistochemical detection to assess the therapeutic efficacy.

Results: The least inhibitive UVB distance and dosage of CLA were selected for the in vivo experiments. CLA effectively weakened the osteogenic inhibitory effect of UVB $(72 \mathrm{~cm})$, significantly improved the activity of alkaline phosphatase (ALP), promoted the formation of mineralized nodules, and alleviated the oxidative damage induced by UVB. CLA also effectively weakened the osteoclast-promoting effect of UVB $(72 \mathrm{~cm})$, inhibited osteoclast formation, and inhibited the inflammatory damage to BMMCs caused by UVB $(72 \mathrm{~cm})$ irradiation. Micro-CT results showed that UVB irradiation could promote bone formation in ovariectomized Sprague-Dawley rats, while CLA could significantly promote bone regeneration. Immunofluorescence assays results showed that CLA alleviated UVB-induced oxidative damage to osteoblasts. The ROS detection results demonstrated that CLA effectively alleviated UVB-induced oxidative damage to BMSCs. Furthermore, Immunohistochemical assays showed that UVB and CLA treatment increased bone density, inhibited osteolytic osteolysis, and enhanced osteogenic activity.

Conclusions: CLA can effectively weaken osteoclast promotion, osteogenic inhibition, and oxidative damage caused by UVB. Combination treatment of UVB and CLA exerts an osteoprotective effect on ovariectomized osteoporotic rats and stimulates osteogenesis. The molecular mechanism of this interaction requires further investigation. 
Keywords: Ultraviolet light B (UVB); conjugated linoleic acid (CLA); osteogenic inhibition; osteoclast promotion; ovariectomized model rats

Submitted Jan 29, 2021. Accepted for publication Mar 25, 2021.

doi: $10.21037 /$ atm-21-934

View this article at: http://dx.doi.org/10.21037/atm-21-934

\section{Introduction}

Osteoporosis is a systemic skeletal disease characterized by a reduction in bone mass. Patients with osteoporosis have increased bone fragility and are susceptible to fractures due to micro-architectural deterioration of bone tissue (1). Data from the World Health Organization (WHO) showed that in Europe, the United States, and Japan alone, approximately 75 million people were living with osteoporosis $(2,3)$. Due to population aging, the risk posed by osteoporotic fractures and their associated costs is rising rapidly (3). Hormone deficiency is known to impair cancellous metaphyseal bone and reduce bone mineral density (BMD) in humans and animals; therefore, estrogen deficiency is regarded as a critical factor in the susceptibility of postmenopausal women to osteoporosis (4). In recent years, this therapy has become a popular therapeutic strategy for postmenopausal osteoporosis and its longterm application can have potential malignant effects on reproductive tissues (5-7). Other medicines that stimulate bone formation or inhibit bone resorption may prevent the progression of bone loss in established osteoporosis. But the above medications can have side effects, including gastrointestinal reactions, cancer, osteonecrosis of the jaw, and reduced skeletal strength (8). In a word, there are efforts to develop new therapeutic schedules with improved efficacy and fewer undesirable side effects to substitute or reduce the medicines currently available.

Exposure of the skin to ultraviolet (UV) light stimulates the synthesis of approximately $90 \%$ of the vitamin D required by humans (9). The clinical impact of vitamin D deficiency on the development of osteoporosis has been demonstrated in numerous clinical investigations. Studies have shown that patients with osteoporosis have lower concentrations of vitamin D than healthy individuals $(10,11)$. A single dose of UV radiation each week can provide an adequate supply of vitamin D (12). In older people, who need a higher ultraviolet B (UVB) intensity than young people to stimulate the synthesis of vitamin D in the skin (13), only very low doses can meet the demand (14). In view of the increasing reluctance among patients to take oral medications, irradiation of the skin with UV light can be used as an alternative treatment to counter vitamin $\mathrm{D}$ deficiency (15). Moreover, this method also has the advantage of not resulting in intoxication $(16,17)$. Recently, several studies have demonstrated that many harmful effects of short-wavelength UVB rays (290-320 nm) can occur through the generation of reactive oxygen species (ROS) (18). The oxidative stress response of cells and tissues involves the induction of oxidative damage in various cellular components, such as membrane lipids, proteins, and DNA, as well as the occurrence of inflammation, immunosuppression, and apoptosis (19). However, the mechanism underlying the effects of UVB in rats with ovariectomy-induced osteoporosis has not been fully elucidated.

One dietary bioactive component that has drawn significant attention over the last 2 decades for its effect on body fat reduction is conjugated linoleic acid (CLA). CLA is a term used to describe a group of geometric and positional isomers of linoleic acid, which was originally identified as an anticancer component found in ground beef (20). Since its discovery, additional biological functions of CLA have been demonstrated. For instance, it can reduce the risk of atherosclerosis, hypertension, and diabetes (21), as well as improve immune function (22), musculoskeletal health, and-most importantly from our perspective-BMD (23). Dietary CLA may help to maintain healthy BMD in postmenopausal women (24). CLA treatment was found to be associated with decreased levels of bone resorption markers, osteoclastogenic genes, and signaling molecules in osteoclastic cell lines $(25,26)$, as well as increased levels of bone formation markers, such as osteocalcin and alkaline phosphatase (ALP), in a murine osteoblastic cell line (27). It has also been reported to increase mineralized bone nodule formation in human osteoblast-like cells (28), and to increase osteoblast differentiation and decrease adipocyte differentiation in human mesenchymal stem cells (29). Given that CLA modulates the activity of prostaglandin E2 (PGE2), it is possible that CLA supplementation could 
reduce bone loss in postmenopausal women and may exert inhibitory effects on osteoclastogenesis. Meanwhile, an ability of CLA to inhibit ROS, haemoxygenase-1, and cyclooxygenase- 2 messenger RNA expression to protect cells from oxidative damage has been reported (30). Currently, it is unclear whether CLA protects against bone loss as a result of the upregulation of osteoblastic factors and bone formation, or as a result of the downregulation of osteoclastic factors and bone resorption. Therefore, this paper was designed to determine the interactions between CLA and UVB and their effects on bone mesenchymal stem cells (BMSCs) and BMMCs to test whether CLA can overcome UVB-induced osteoclast promotion, osteogenic inhibition, and oxidative damage. We also screened a therapeutic scheme combining UVB and CLA, and examined the effects of this treatment in ovariectomized model rats with osteoporosis. We present the following article in accordance with the ARRIVE reporting checklist (available at http://dx.doi.org/10.21037/atm-21-934).

\section{Methods}

\section{Reagents and antibodies}

CLA has 2 main isomers, cis-9, trans-11 $(\mathrm{c} 9, \mathrm{t} 11)$ and trans-10, cis-12 (t10,c12). Cell experiments were performed with CLA containing c9,t11 (Sigma-Aldrich, 16413,Shanghai,China) and t10,c12 (Sigma-Aldrich, 04397) in a 50:50 ratio. Animal experiments were also carried out using CLA in a 50:50 ratio (customized by Qingdao Aohai Biological). Hoechst and Cell Counting Kit-8 (CCK-8) C0037 were obtained from Beyotime Biotechnology (Shanghai, China). Receptor activator of nuclear factor- $\mathrm{\kappa B}$ ligand (RANKL) 315-11C-100 and recombinant murine macrophage colony-stimulating factor (M-CSF) 315-0210 were obtained from PeproTech (Rocky Hill, NJ, USA). Anti-CD90 antibody ab225 was purchased from Abcam (Cambridge, UK). Cy3-labeled anti-mouse secondary antibody SA00009-1 was obtained from Proteintech (Chicago, USA). A ROS test kit CA1410 and osteoclast anti-tartaric-resistant acidic phosphatase (TRAP) staining solution G1492 were obtained from Solarbio (Beijing, China). A Cell Navigator F-Actin Labeling Kit Green Fluorescence 22661 was obtained from AAT Bioquest. Rat interleukin (IL)-6 E-EL-R0015c, tumor necrosis factor (TNF)- $\alpha$ E-EL-R2856c, and IL-10 E-EL-R0016c enzymelinked immunosorbent assay (ELISA) kits were supplied by Elabscience. Rat serum ALP was detected using a Zecheng
Biotechnology instrument. Rat C-terminal telopeptide (CTX) E-EL-R1405c, parathyroid hormone-C (PTH-C) E-EL-R0535c, 25-hydroxy vitamin D3 (25-HVD3) E-EL$0015 \mathrm{c}$, calcium, and osteocalcin (OC) E-EL-R0243c were obtained from Elabscience. Superoxide dismutase (SOD) detection kits were supplied by Nanjing Jiangcheng Bioengineering Institute (Nanjing, China). An ultraviolet radiation $\mathrm{B}$ (UVB) device was purchased from Philips (Netherlands). All chemicals and reagents used were of analytical grade.

\section{Cells and cell culture}

For BMSC preparation, bone marrow was collected from rat femurs and tibias in a sterile environment and transferred into centrifuge tubes. The centrifuge tubes containing the bone marrow were then centrifuged at $1,500 \mathrm{rpm}$ for 20 minutes. Precipitated cells were suspended in Dulbecco's Modified Eagle Medium (DMEM) containing $15 \%$ fetal bovine serum (FBS) and penicillin-streptomycingentamicin. After 7 days, red blood cells were washed with phosphate-buffered saline (PBS), and fresh medium was added. For BMMC preparation, bone marrow was collected from rat femurs and tibias in a sterile environment. To stratify the bone marrow components according to density, BMMCs were isolated via density gradient centrifugation with Cellgro (Mediatech). After centrifugation, some red blood cells (non-nucleated cells) and plasma were isolated and removed, following which BMMCs were collected and washed with PBS. Finally, the cells were cultured in Minimum Essential Medium $\alpha(\alpha-M E M)$ containing $15 \%$ FBS, penicillin-streptomycin-gentamicin, and M-CSF.

\section{Osteoporotic rat model establishment}

Female Sprague-Dawley (SD) rats (age, 3 months old; weight, $225 \mathrm{~g}$ ) were obtained from Kavans (Changzhou, China) and kept at room temperature under pathogenfree conditions. All rats were acclimated to the laboratory environment for 1 week before the experiments were performed. Experiments were performed under a project license (No. 2018-52) granted by the Ethics Committee of the First Affiliated Hospital of Soochow University, in compliance with the institutional guidelines for the care and use of animals. After 7 days of acclimatization, the rats underwent ovariectomy or a sham operation. Specifically, the ovariectomized (OVX) rats were anesthetized with pentobarbital sodium ( $40 \mathrm{mg} / \mathrm{kg}$ bw, intraperitoneally), and 
the ovaries were removed bilaterally. For the sham-operated control group, the operation was performed in the same manner but to only expose the ovaries.

\section{Grouping, treatment, and specimen collection of animals}

After the OVX rat model of osteoporosis had been established successfully, the rats were randomly designated to 1 of the following 6 groups: control group, OVX group, OVX + UVB group, OVX + UVB + CLA low-dosage (OVX + UVB + 1CLA) group, OVX + UVB + CLA mediumdosage (OVX + UVB + mCLA) group, OVX + UVB + CLA high-dosage (OVX + UVB + hCLA) group. Rats in OVX + UVB groups were treated with $311 \mathrm{~nm}$ UVB once a day for 2 hours. Rats in the OVX + UVB + 1CLA group were treated daily with CLA $1.4 \mathrm{mmol} / \mathrm{kg}$ intragastrically. Rats in the OVX + UVB + mCLA group were treated daily with CLA $2.8 \mathrm{mmol} / \mathrm{kg}$ intragastrically. Rats in the OVX + UVB + hCLA group were treated daily with CLA $4.2 \mathrm{mmol} / \mathrm{kg}$ intragastrically. At the end of treatment, the rats in all groups were weighed and sacrificed by cervical dislocation, and the femur bones were dissected.

Each rat was individually placed in a metabolic cage without food for 24 hours before sacrifice. After laparotomy under anesthesia with pentobarbital sodium (intraperitoneal injection, $40 \mathrm{mg} / \mathrm{kg}$ ), blood samples were collected from the rats through abdominal aortic puncture. After that, serum specimens were harvested through centrifugation (2,000 rpm for 20 minutes). For further testing and analysis, the samples were stored at $-20{ }^{\circ} \mathrm{C}$. Femurs and the $4^{\text {th }}$ lumbar (L4) vertebrae of the rats were dissected, wrapped with wet gauze to prevent dehydration. Then, it was stored at $-20{ }^{\circ} \mathrm{C}$ for BMD measurement, pathological analysis, and biomechanical testing.

\section{Cytotoxicity assay}

For the cytotoxicity assay, BMSCs or BMMCs were harvested and plated. After 24 hours, non-adherent cells were re-plated at a density of 20,000 cells/well in $\alpha-M E M / 15 \%$ FBS/5\% M-CSF culture medium. To determine cell viability, cells were exposed to UVB radiation at different distances or various concentrations of CLA, and the plates were incubated for a further 24 hours (BMSCs) or 48 hours (BMMCs). Then, $20 \mu \mathrm{L}$ of CCK-8 solution was added into each well 2 hours before culture termination, and the optical density (OD) at $450 \mathrm{~nm}$ was detected with a microplate spectrophotometer (Thermo MK3, Thermo Fisher, USA).

\section{Micro-CT analysis}

Upon the completion of treatment, the rats were euthanized. Rat femora were dissected, cleaned of all soft tissues, and stored in a $10 \%$ formalin solution in preparation for analysis. Then, in vitro micro-CT (Bruker skyscan1176, USA) was applied to obtain microarchitectural bone parameters. Before scanning, the femora were put into a sample holder which can fit up to 8 femora per scan, and measurement protocols were created to define parameters such as source of energy, intensity, and filter. The source of energy and intensity selected were $70 \mathrm{kVp}$ and $114 \mu \mathrm{A}$, respectively. A $0.5-\mathrm{mm} \mathrm{Al}$ filter was utilized. To obtain the best images possible, a high resolution of $10 \mu \mathrm{m}$ was set. Trabecular bone parameters were obtained from the distal end of the femur. The number of slices was set to approximately 200 slices, and the chosen region of interest (ROI) was at the metaphyseal area, located approximately $1.5 \mathrm{~mm}$ below the epiphyseal growth plate, extending $2.0 \mathrm{~mm}$ in the proximal direction. The selected region has a rich blood supply and high bone turnover activity. Trabecular bone was selected because it has a greater surface-to-volume ratio and is more metabolically active than cortical bone (31). This analysis involved a three-dimensional morphometric evaluation comprising both metric and non-metric bone structural parameters. The measurement of structural parameters is similar to the parameters of tissue morphology analysis, and includes bone volume fraction (BV/TV), trabecular thickness (Tb.Th), trabecular separation (Tb.Sp), and trabecular number ( $\mathrm{Tb}$. $\mathrm{N})$. The non-metric parameters measured were connectivity density (Conn.dens), structural model index (SMI), and degree of anisotropy (DA).

\section{Biochemical analysis of rat serum specimens}

Serum levels of calcium (Ca), phosphorus (P), and ALP were measured with an automatic Zecheng biochemical analyzer (Nanjing, China) using a diagnostic reagent kit. Serum OC, CTX, PTH-C, 25-HVD3, SOD, IL-6, TNF- $\alpha$, and IL-10 concentrations were determined using rat ELISA kits (Elabscience, CA, USA). All procedures were conducted in adherence to the manufacturer's instructions.

\section{Immunobistochemical and immunofluorescence assays}

BMSCs or BMMCs were seeded at a density of 20,000 cells per plate and exposed to UVB radiation at different distances and/or various concentrations of CLA. The 


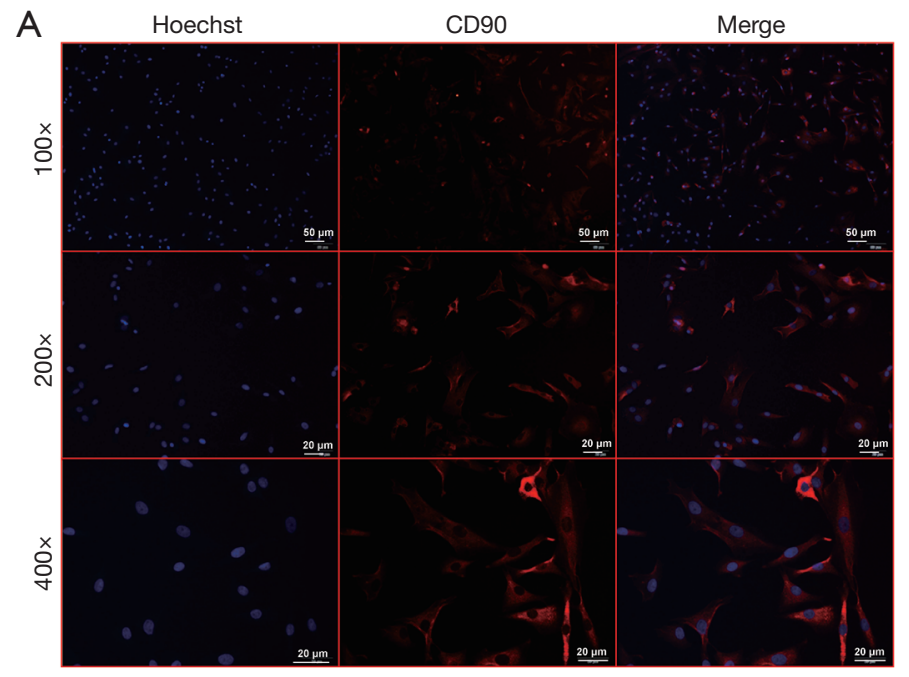

B
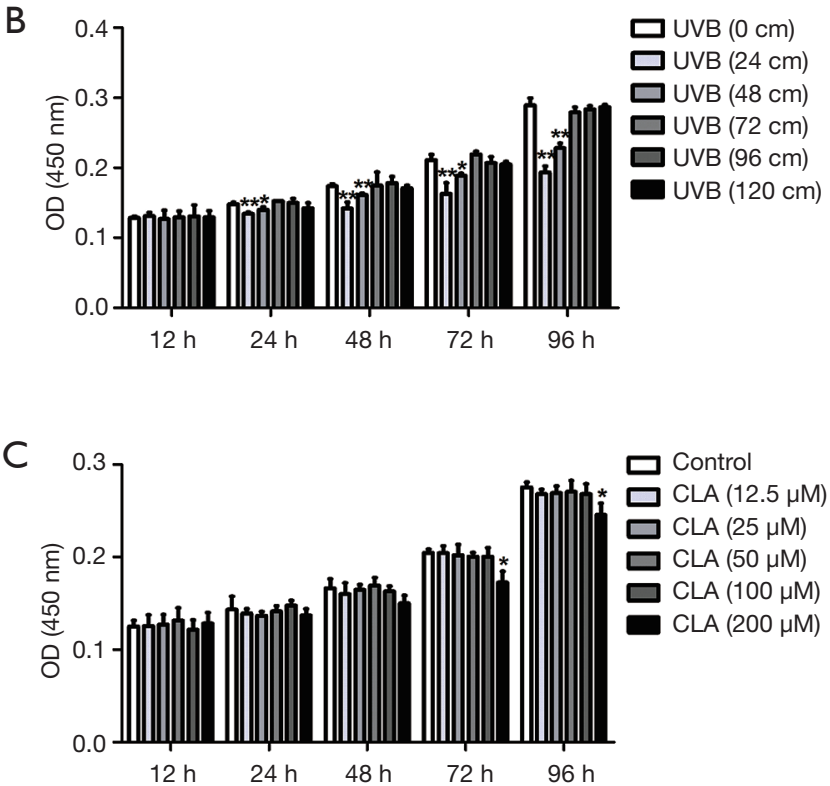

Figure 1 After treatment with UVB or CLA, BMSCs were subjected to immunofluorescence staining and CCK-8 detection. (A) Staining of the BMSC marker CD90 in rat bone tissue. Images were captured at $\times 100, \times 200$, and $\times 400$ magnification, $(n=3)$. (B) Effects of UVB treatment at different distances on the proliferation of BMSCs in vitro $(\mathrm{n}=3)$. BMSC proliferation was assessed after stimulation with UVB for 24 hours. (C) Effects of treatment with CLA at different dosages on the proliferation of BMSCs in vitro $(\mathrm{n}=3)$. BMSC proliferation was assessed after stimulation with CLA for 24 hours. Data are shown as the mean \pm standard deviation (SD). Compared with control, ${ }^{*} \mathrm{P}<0.05$, ${ }^{* *} \mathrm{P}<0.01$. BMSC, bone mesenchymal stem cell; UVB, ultraviolet B; CLA, conjugated linoleic acid.

BMSCs and BMMCs were incubated at $37^{\circ} \mathrm{C}$ for 24 hours and 48 hours, respectively. Then, the cells were stained with anti-CD90 antibody, ALP dye, Alazarin red S, DCFHDA (ROS dye), TRAP stain, phalloidin (F-actin dye), and Hoechst 33342 (nuclear dye), and images were taken with a confocal microscope at $100 \times$ magnification.

Immunohistochemical assays of rat femur were conducted on frozen tissue sections. The sections were heated in an ethylenediaminetetraacetic acid (EDTA) antigen retrieval buffer for antigen repair and incubated in aqueous hydrogen peroxide solution to quench endogenous peroxidase. After incubation with primary then secondary antibody, the sections were incubated with diaminobenzidine to colour the antigens. Following that, the sections were successively incubated in hematoxylin solution, hematoxylin differentiation solution, and haematoxylin blue solution to counterstain the nuclei. Finally, the sections were air-dried and sealed with neutral gum.

\section{Statistical analysis}

GraphPad Prism 7.0 software was used for all statistical analyses. One-way analysis of variance (ANOVA) was employed for comparisons of more than 2 group means. Significance in differences between groups was indicated by ${ }^{*} \mathrm{P}<0.05,{ }^{* *} \mathrm{P}<0.01$, and ${ }^{* * *} \mathrm{P}<0.001$.

\section{Results}

\section{$C L A$ alleviated oxidative damage to osteoblasts caused by UVB}

\section{Identification of rat BMSCs and dosage screening of} UVB and CLA

As shown in Figure 1A, cell nuclei were marked in blue with Hoechst and CD90 was marked in red with antibody. After merging, CD90 could be seen on the surface of BMSCs. Figure $1 B$ shows that the growth of BMSCs was significantly inhibited when the distance of UVB $(311 \mathrm{~nm})$ irradiation was $24 \mathrm{~cm}$ and $48 \mathrm{~cm}$ (irradiation time $\geq 24$ hours). Consequently, $\geq 72 \mathrm{~cm}$, as a non-inhibitive distance, was selected for UVB treatment in subsequent experiments. As shown in Figure 1C, when the CLA dose was $\leq 100 \mu \mathrm{M}$, BMSC proliferation was not affected, but the growth rate of 
BMSCs was inhibited by a CLA dosage of $>200 \mu M$. Thus, $\leq 100 \mu \mathrm{M}$, as the non-inhibitive dosage, was selected for CLA treatment in subsequent experiments.

\section{UVB irradiation promoted BMSC osteogenesis and} UVB had inhibitive effects at a distance of $72 \mathrm{~cm}$ As shown in Figure $2 \mathrm{~A}$, the ALP activity in BMSCs increased significantly after osteogenic induction and UVB irradiation, which indicated that osteogenic differentiation was promoted. However, Figure $2 A$ also shows that from a distance of $72 \mathrm{~cm}, \mathrm{UVB}$ treatment had a less significant effect on the promotion of ALP activity in BMSCs than it did at longer distances. As displayed in Figure 2B, on days 14 and 21 after induction, compared with the osteogenic induction group, the level of ALP was lower in the 72-cm UVB irradiation group, indicating that osteogenic differentiation was slightly inhibited by irradiation at this distance. As revealed in Figure $2 C$, on days 14 and 21 after induction, compared with the osteogenic induction group, there were fewer mineralized nodules in the $72-\mathrm{cm}$ UVB irradiation group, indicating that osteogenic differentiation was inhibited by irradiation at this distance. Also, Figure $2 D$ shows that ROS production in BMSCs was increased after UVB treatment, especially in the $72-\mathrm{cm}$ UVB treatment group, which suggested that UVB delivered from this distance caused oxidative damage to BMSCs.

The above results demonstrated that when the irradiation distance of UVB was $\geq 96 \mathrm{~cm}$, there was no significant inhibition of osteogenic differentiation of BMSCs. When the UVB irradiation distance was $72 \mathrm{~cm}$, ALP activity and other related results evidenced slight inhibition of BMSC osteogenic differentiation as well as the induction of oxidative damage.

\section{CLA alleviated UVB-induced oxidative damage to osteoblasts}

Figure $3 A$ shows that on day 14 of osteogenic induction, ALP activity was slightly decreased in the BMSCs in the 72-cm UVB irradiation group compared with osteogenic induction alone group. However, CLA weakened the osteogenic inhibition induced by UVB and significantly improved ALP activity. The results of ALP staining also showed that CLA effectively weakened the osteogenic inhibition induced by UVB at $72 \mathrm{~cm}$ (Figure 3B), and significantly improved ALP activity. Similarly, the results of alizarin red staining revealed that CLA effectively weakened UVB $(72 \mathrm{~cm})$-induced osteogenic inhibition and significantly promoted the production of mineralized nodules (Figure 3C). Furthermore, the ROS detection results demonstrated that CLA effectively alleviated UVBinduced oxidative damage to BMSCs (Figure 3D).

The above results demonstrated that CLA effectively weakened the osteogenic inhibitory effects of UVB delivered from a distance of $72 \mathrm{~cm}$, significantly elevated ALP activity, promoted mineralized nodule formation, and alleviated oxidative damage induced by UVB $(72 \mathrm{~cm})$.

\section{$C L A$ inbibited UVB-induced osteoclast formation on BMMCs}

Dosage screening of UVB and CLA using rat BMMCs As shown in Figure $4 A$, cell nuclei were marked in blue with Hoechst and CD90 was marked in red with antibody. After merging, CD90 could be seen on the surface of BMMCs. When the UVB $(311 \mathrm{~nm})$ irradiation distance was $24 \mathrm{~cm}$ or $48 \mathrm{~cm}$ and the irradiation time reached or exceeded 48 hours, BMMC proliferation was significantly inhibited (Figure $4 B$ ). Therefore, $\geq 72 \mathrm{~cm}$, as a non-inhibitive distance was selected for UVB treatment in subsequent experiments. The results of CCK- 8 assay showed that BMMC proliferation was not inhibited by CLA at a dosage of $\leq 100 \mu \mathrm{M}$ but was inhibited to some extent after treatment with CLA $\geq 200 \mu \mathrm{M}$ (Figure 4C). Therefore, CLA $\leq 100 \mu \mathrm{M}$, as the non-toxic dose, was selected for the subsequent experiments.

\section{UVB irradiation induced osteoclast formation on rat BMMCs}

The results of TRAP staining (Figure $5 A$ ) showed that UVB treatment from a distance of $72 \mathrm{~cm}$ promoted osteoclastic differentiation of BMMCs, thereby enhancing TRAP activity. The F-actin staining results (Figure 5B) also revealed that osteoclastic differentiation of BMMCs was significantly promoted under 72 -cm UVB treatment.

\section{CLA inhibited UVB-induced osteoclast formation on BMMCs}

The results of TRAP staining (Figure $6 A$ ) showed that CLA could effectively weaken the promoting effects of UVB $(72 \mathrm{~cm})$ on osteoclastic differentiation and significantly inhibit TRAP activity in a dose-dependent manner. F-actin staining (Figure 6B) also demonstrated that CLA dosedependently weakened the UVB-induced promotion of osteoclastic differentiation and significantly inhibited osteoclast formation. The results of ELISA assay (Figure 6C) showed that CLA effectively inhibited the UVB- 

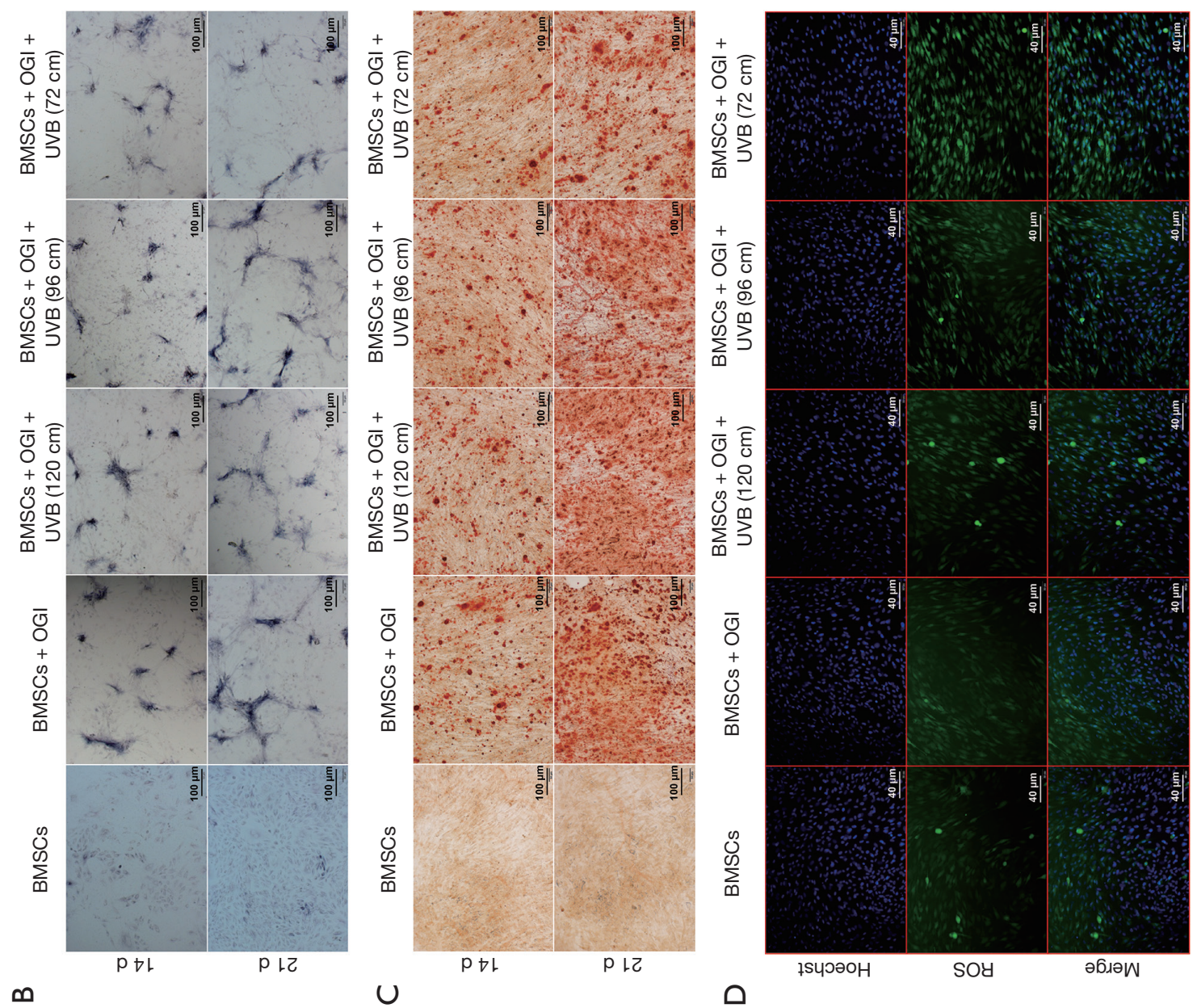

$\infty$

$$
\cup
$$

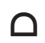

łsчәәон

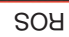

әБцәW
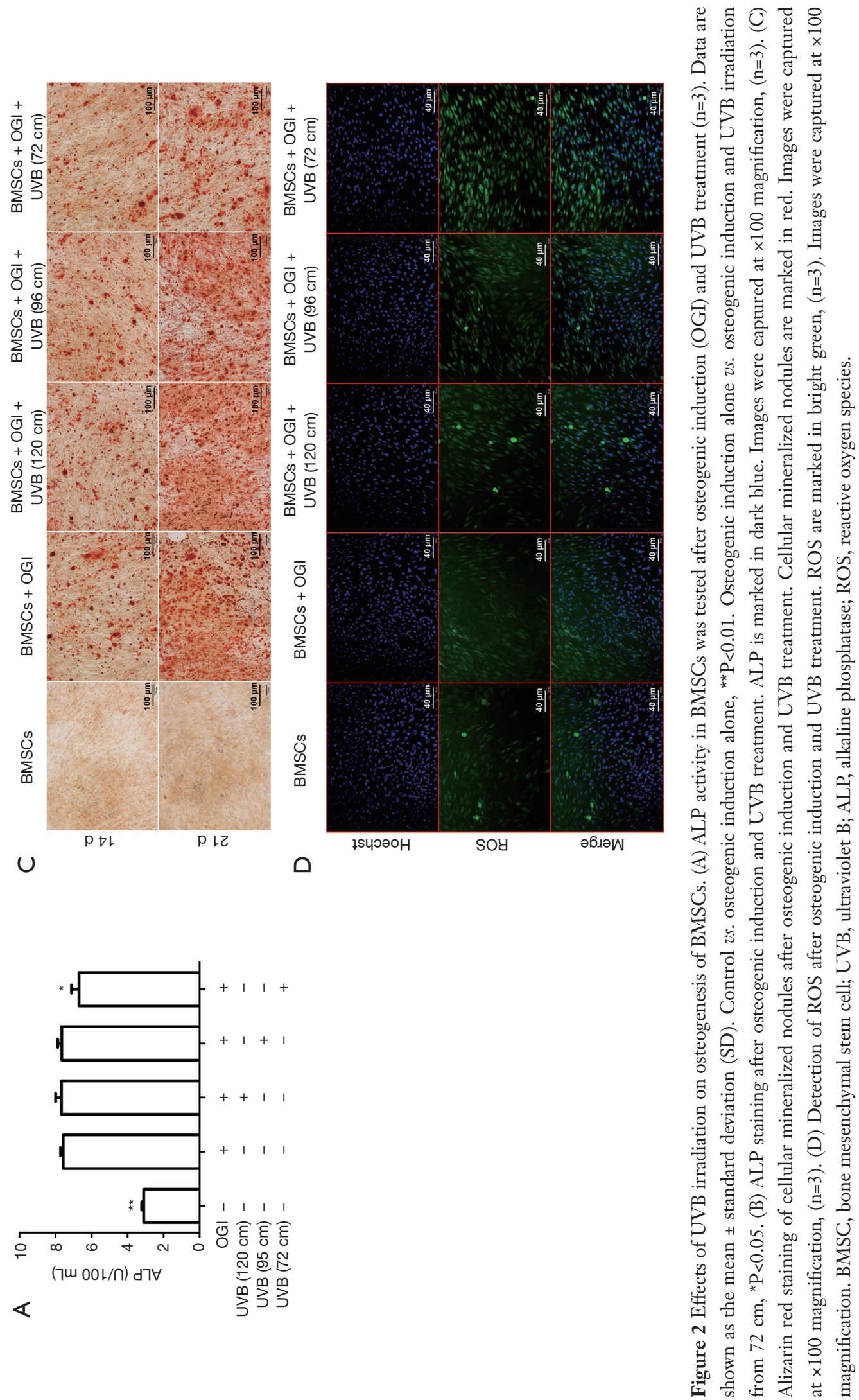

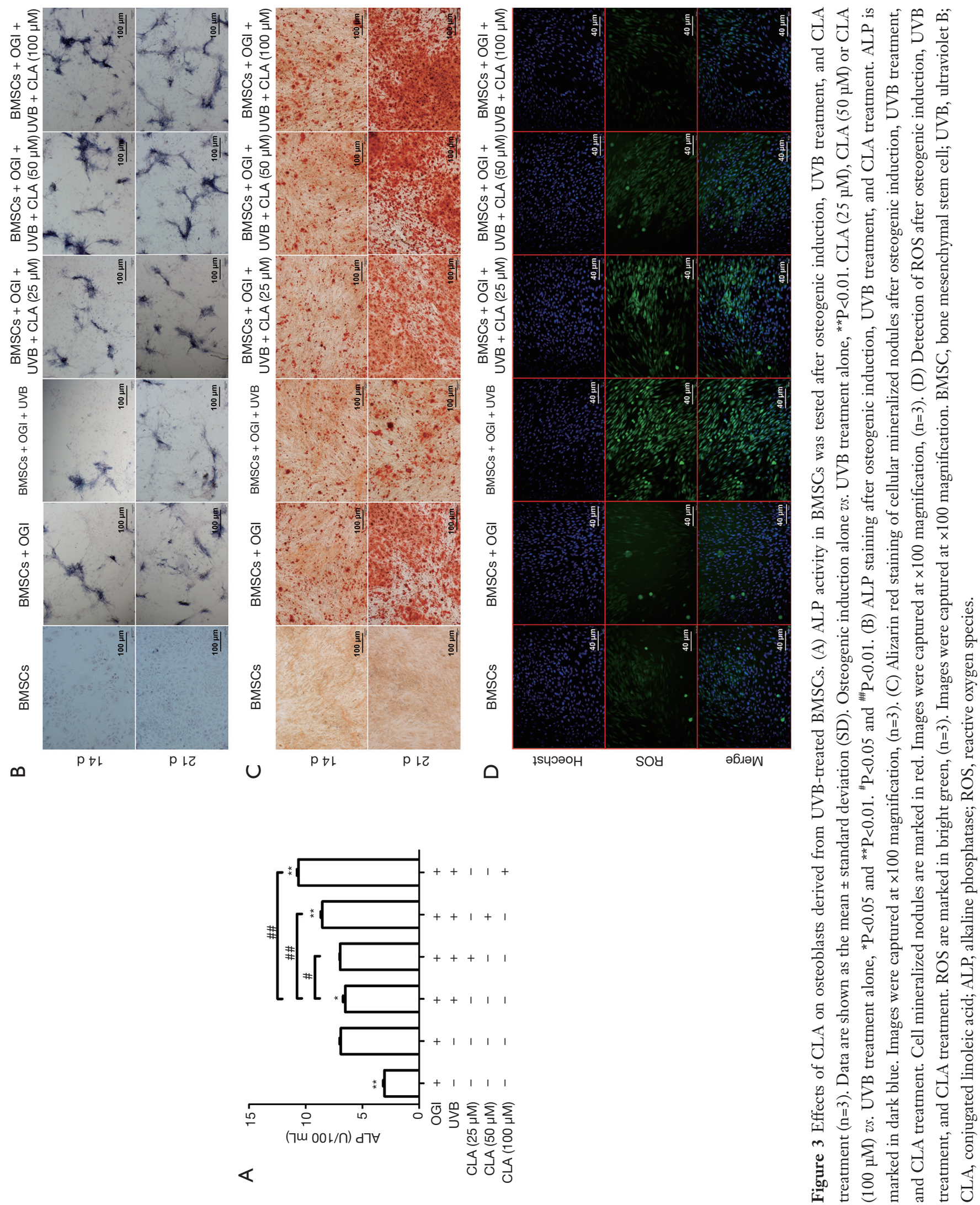

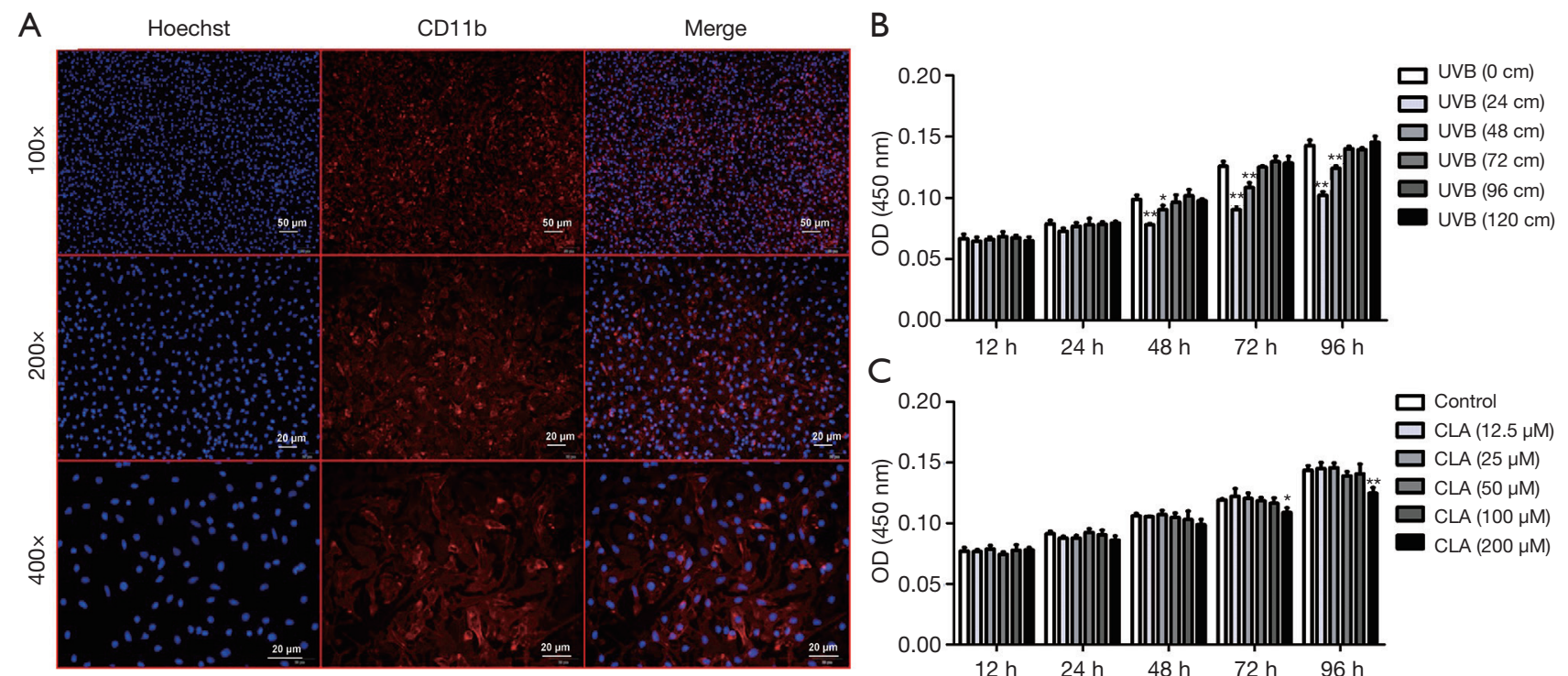

Figure 4 After treatment with UVB or CLA, BMMCs were subjected to immunofluorescence staining and CCK-8 detection. (A) staining of the BMMCs marker CD90 in rat bone tissue $(n=3)$. Images were captured at $\times 100, \times 200$, and $\times 400$ magnification. (B) Effects of treatment with UVB at different distances on the proliferation of BMMCs in vitro $(\mathrm{n}=3)$. BMMC proliferation was assessed after stimulation with UVB. (C) Effects of CLA at different dosages on the proliferation of BMMCs in vitro ( $\mathrm{n}=3)$. BMMC proliferation was assessed after stimulation with CLA. Data are shown as the mean \pm standard deviation $(\mathrm{SD})$. Compared with control, ${ }^{*} \mathrm{P}<0.05,{ }^{* *} \mathrm{P}<0.01$. BMMC, bone marrow mononuclear cell; UVB, ultraviolet B; CLA, conjugated linoleic acid.

induced expression of inflammatory factors IL-6 and TNF- $\alpha$ in BMMCs, while promoting the production of anti-inflammatory factor IL-10. Together, these results showed that CLA could effectively weaken the osteoclastpromoting effect of UVB $(72 \mathrm{~cm})$, inhibit osteoclast formation, and inhibit the inflammatory effects of UVB $(72 \mathrm{~cm})$ on BMMCs.

\section{$U V B$ and $C L A$ promoted bone formation and regeneration in $O V X S D$ rats}

UVB promoted bone formation and CLA significantly promoted bone regeneration in OVX SD rats

The results of micro-CT (Figure 7) showed that UVB irradiation promoted bone formation in OVX SD rats, while CLA significantly promoted bone regeneration.

The effects of ovariectomy and treatment on the body weights and intakes of the rats

We have established an osteoporosis model to measure the intake and weight of rats (Figure $8 A$ ). After ovariectomy, the intakes of the model rats increased significantly, and their body weights also showed a rapid increase (Figure 8B).
Meanwhile, the body weights of UVB- and CLA-treated rats increased slowly, and their intakes decreased (Figure $8 B, C)$. The intakes and body weights of the UVB- and CLA-treated rats were close to those of the control rats.

\section{UVB and CLA treatment decreased bone absorption and increased bone formation}

With the continuation of UVB and CLA treatment, the serum concentrations of ALP (Figure 9A), phosphorus (Figure 9B), CTX (Figure 9C), and PTH-C (Figure 9D) of $\mathrm{SD}$ rats decreased significantly compared with the model group, which indicated that bone absorption was inhibited.

The serum concentrations of 25-HVD3 (Figure 9E), calcium (Figure 9F), and osteocalcin (Figure 9G) of SD rats increased significantly compared with the model group as UVB and CLA treatment continued. These results indicated that UVB and CLA could promote the synthesis of vitamin $\mathrm{D}$ as well as calcium accumulation, and osteoblast activation, so as to effectively promote osteocalcin, bone formation, and the alleviation of osteoporosis. UVB irradiation aggravated oxidative damage, which was evidenced by significantly lower levels of serum SOD in treated rats than 


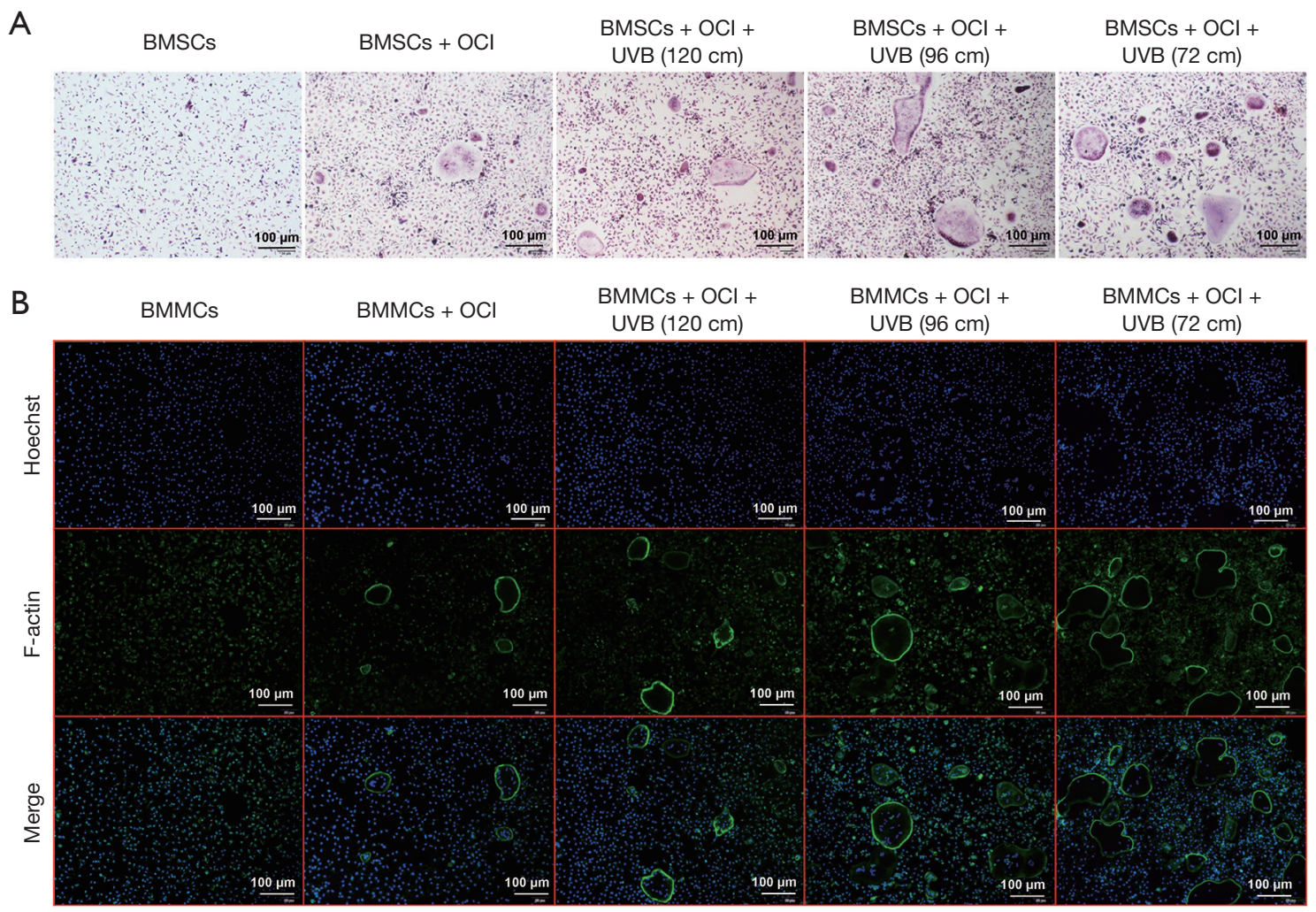

Figure 5 TRAP staining and F-actin staining of BMMCs treated with UVB at different distances. (A) TRAP staining of BMMCs treated with UVB at different distances. TRAP is marked in purple ( $\mathrm{n}=3$ ). Images were captured at $\times 100$ magnification. (B) F-actin staining of BMMCs after treatment with UVB at different distances and osteoclast induction (OCI), ( $\mathrm{n}=3)$. Cell nuclei are marked in blue. F-actin is marked in green. Images were captured at $\times 100$ magnification. TRAP, tartaric-resistant acidic phosphatase; BMMC, bone marrow mononuclear cell; UVB, ultraviolet B.

in the model group (Figure 9H). In contrast, CLA treatment significantly elevated the levels of SOD in rat serum, which effectively alleviated the oxidative damage caused by UVB irradiation.

\section{UVB and CLA treatment increased bone density,} inhibited osteolytic osteolysis, and enhanced osteogenic activity

Hematoxylin-eosin staining (Figure 10A) revealed that the OVX model rats had loose bone tissues, with fractured and poorly organized trabeculae, and enlargement of the marrow cavity. Meanwhile, UVB- and CLA-treated rats had more trabeculae with fewer fractures, implying that the combination treatment had a repairing effect. The results of TRAP staining (Figure 10B) showed that TRAP-positive areas of bone tissue from OVX rats were significantly increased, but were reduced after UVB irradiation and CLA treatment, suggesting that UVB and CLA treatment inhibited osteolytic osteolysis in vivo. The results of alizarin red staining (Figure 10C) showed that positively stained areas of bone tissue from OVX rats were significantly decreased, while after UVB and CLA treatment, positively stained areas increased, suggesting that this therapeutic regimen enhanced osteogenic activity and promoted bone regeneration in vivo. These results were consistent with the results of micro-CT.

\section{Discussion}

In the present investigation, we have shown that treatment with UVB and CLA affects bone formation and regeneration in OVX osteoporosis model rats. OVX SD rats were treated with UVB from a distance of $72 \mathrm{~cm}$ and different doses of CLA. As treatment continued, the levels of bone resorptionrelated key factors, including ALP, phosphorus, CTX, and PTH-C, decreased, while those of bone formation- 
A

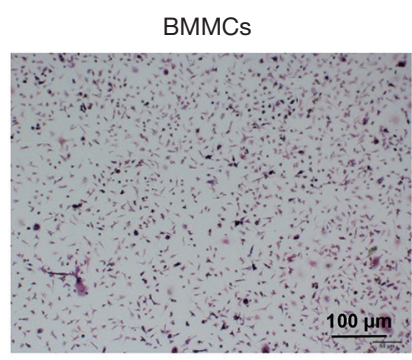

$\mathrm{BMMCs}+\mathrm{OCl}+$

UVB + CLA $(25 \mu \mathrm{M})$

B

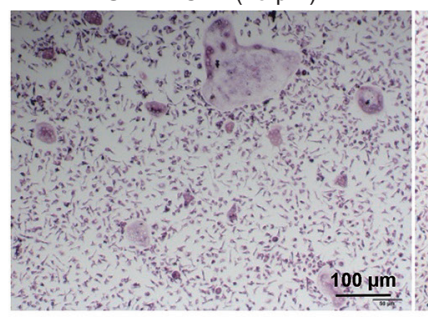

$\mathrm{BMMCs}+\mathrm{OCl}$

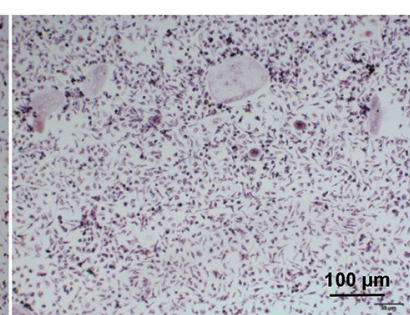

$\mathrm{BMMCs}+\mathrm{OCl}+\mathrm{UVB}$

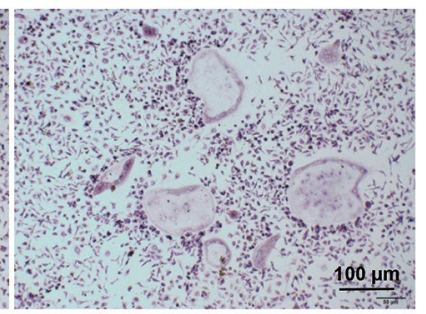

$\mathrm{BMMCs}+\mathrm{OCl}+$ UVB + CLA $(100 \mu \mathrm{M})$ UVB + CLA $(50 \mu \mathrm{M})$
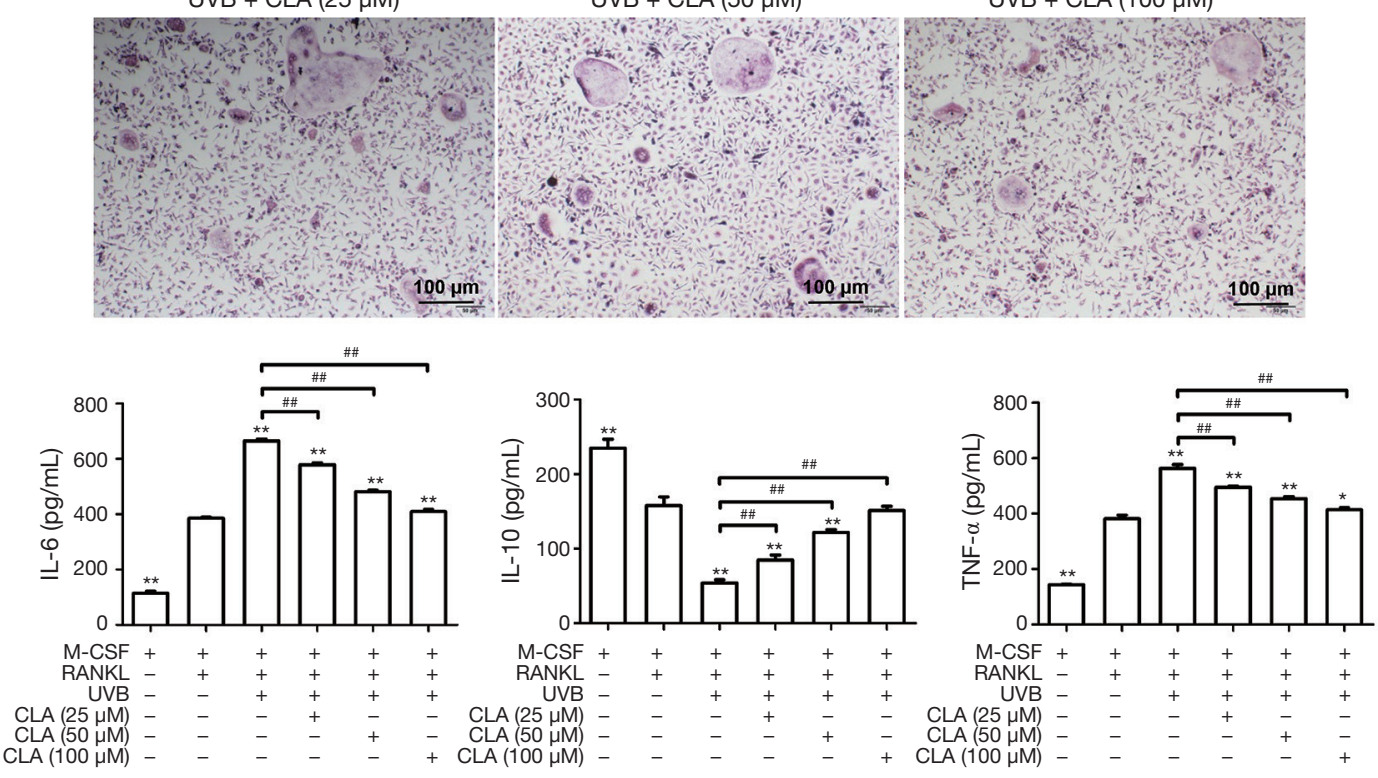

C

C

$\mathrm{BMMCs}+\mathrm{OCl}+\mathrm{BMMCs}+\mathrm{OCl}+\mathrm{BMMCs}+\mathrm{OCl}+$

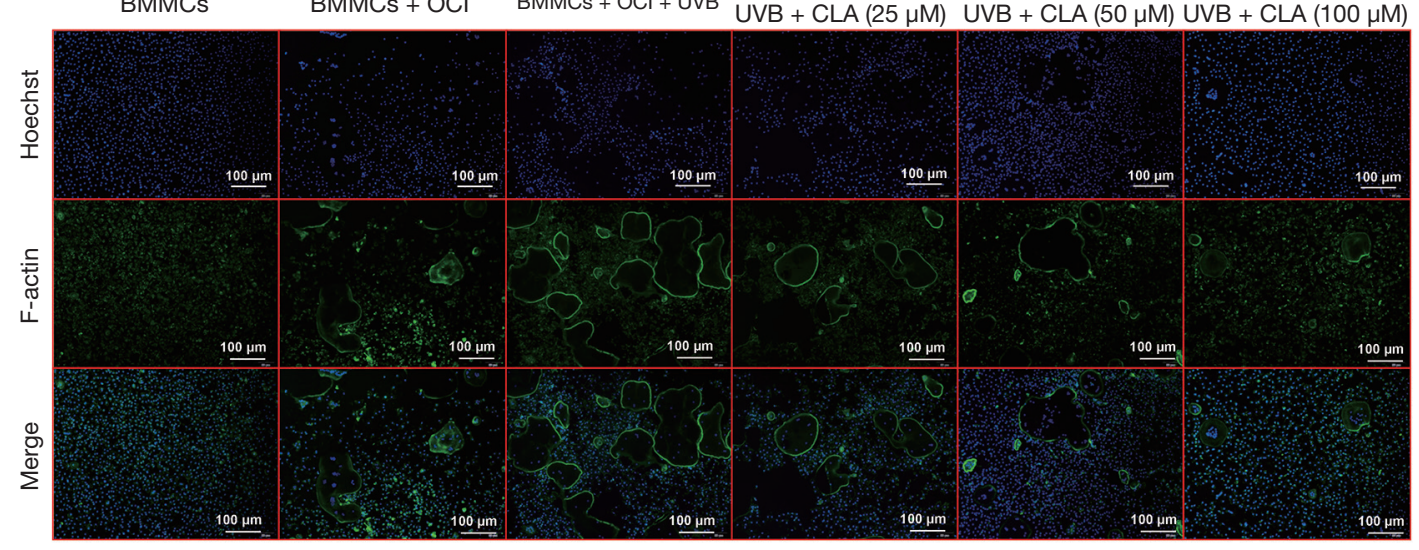

Figure 6 TRAP staining, F-actin staining, and ELISA assay of UVB- and CLA-treated BMMCs. (A) TRAP staining of BMMCs after osteoclast induction, UVB treatment, and CLA treatment. TRAP is marked in purple $(\mathrm{n}=3)$. Images were captured at $\times 100$ magnification. (B) Culture medium supernatant ELISA assay of BMMCs after osteoclast induction, UVB treatment, and CLA treatment (n=3). (C) F-actin staining of BMMCs after osteoclast induction, UVB treatment, and CLA treatment. Cell nuclei are marked in blue, ( $\mathrm{n}=3$ ). F-actin is marked in green. Images were captured at $\times 100$ magnification. Data are shown as the mean \pm standard deviation (SD). Compared with M-CSF- and RANKL-treated BMMC group, ${ }^{*} \mathrm{P}<0.05$ and ${ }^{* *} \mathrm{P}<0.01 .{ }^{*} \mathrm{P}<0.05$ and ${ }^{\# \#} \mathrm{P}<0.01$. TRAP, tartaric-resistant acidic phosphatase; ELISA, enzymelinked immunosorbent assay; BMMC, bone marrow mononuclear cell; UVB, ultraviolet B; CLA, conjugated linoleic acid. 


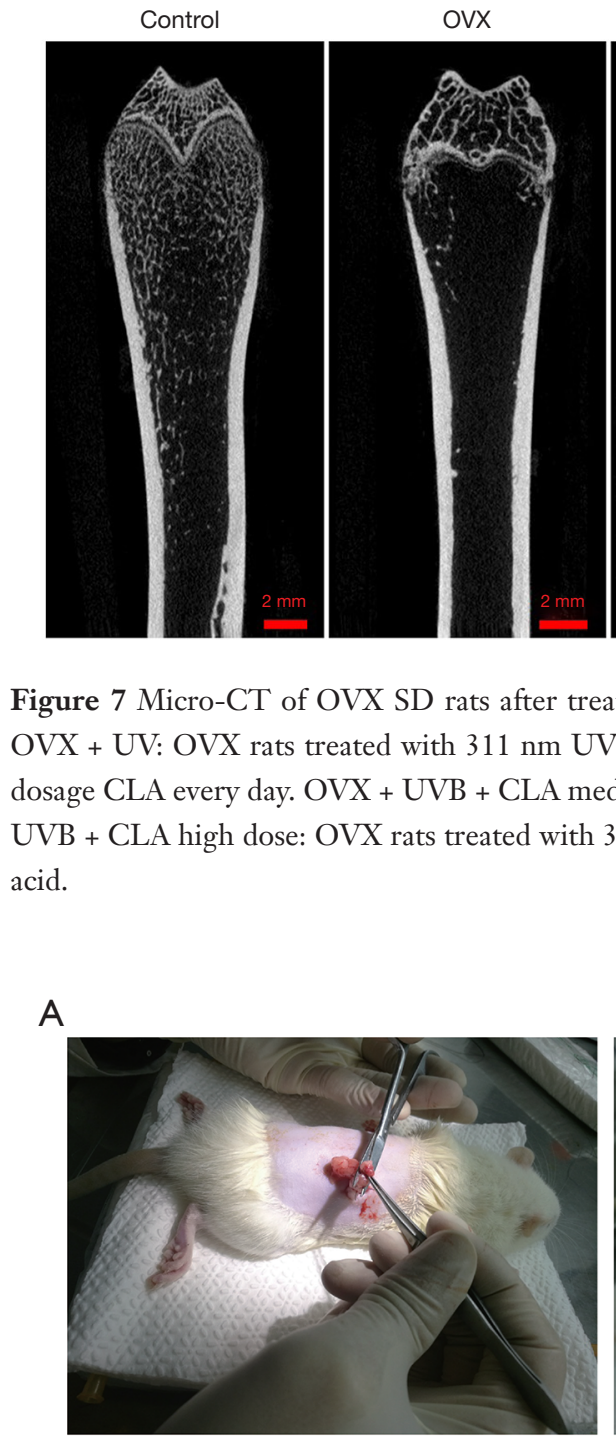

OVX + UVB

OVX + UVB + ICLA

OVX + UVB + mCLA

OVX + UVB + hCLA

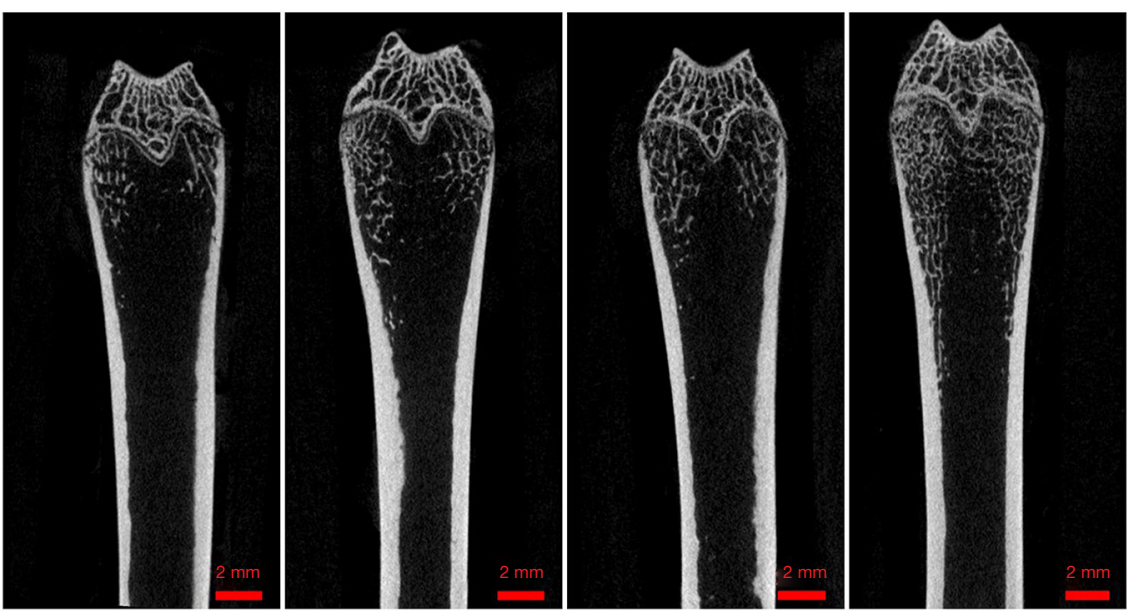

Figure 7 Micro-CT of OVX SD rats after treatment with UVB and CLA, (n=5).Control: sham-operated rats. OVX: ovariectomized rats. OVX + UV: OVX rats treated with $311 \mathrm{~nm}$ UVB every day. OVX + UVB + CLA low dose: OVX rats treated with $311 \mathrm{~nm}$ UVB and lowdosage CLA every day. OVX + UVB + CLA medium dose: OVX rats treated with $311 \mathrm{~nm}$ UVB and medium-dosage CLA every day. OVX + UVB + CLA high dose: OVX rats treated with $311 \mathrm{~nm}$ UVB and high-dosage CLA every day. UVB, ultraviolet B; CLA, conjugated linoleic acid.
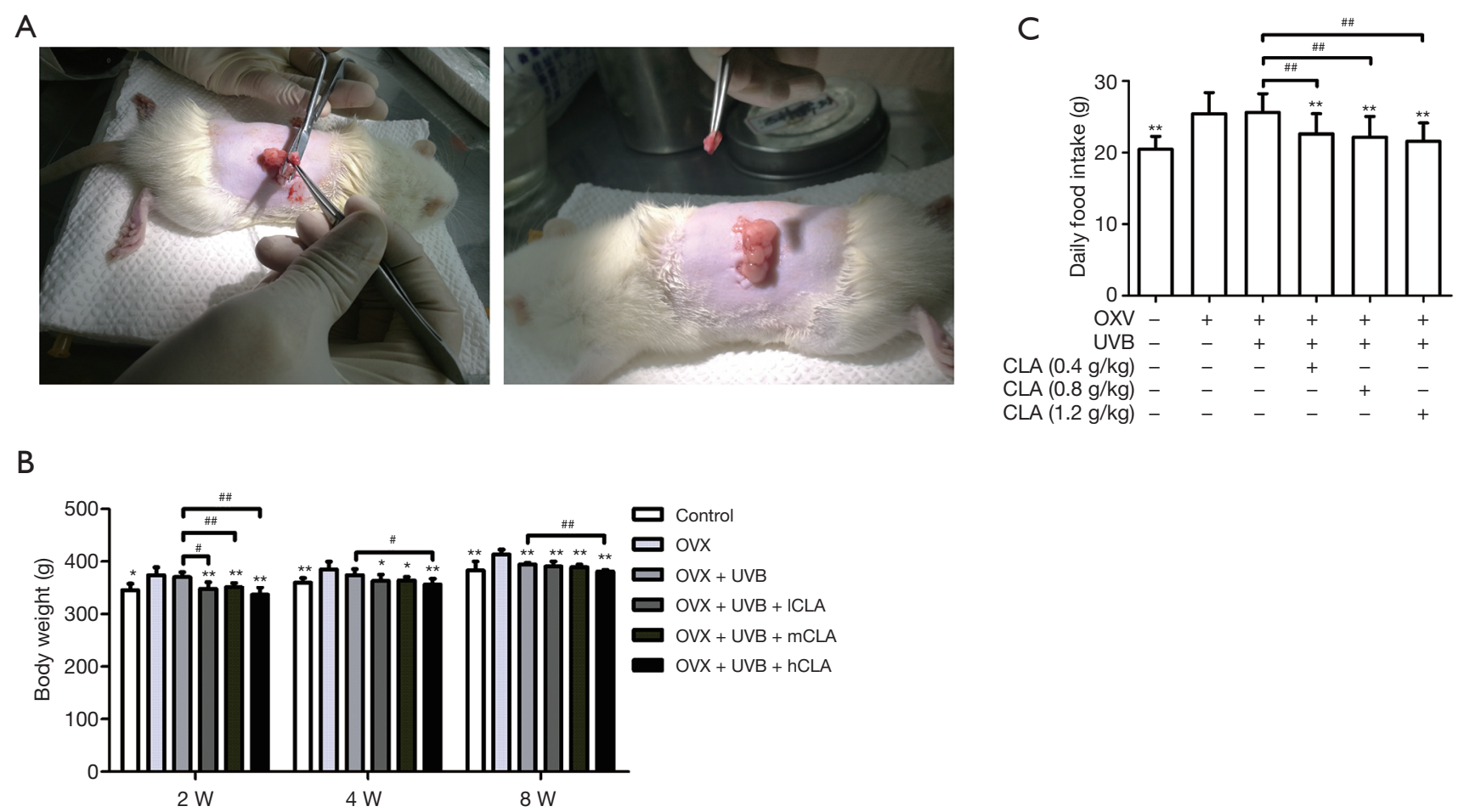

Figure 8 Establishment of the osteoporosis model, and intakes and body weights of rats. (A) Establishment of ovariectomized osteoporosis model rats. (B) The body weights of the rats were monitored every 2 weeks during treatment, $(\mathrm{n}=5)$. (C) The intakes of the rats were monitored during treatment, $(\mathrm{n}=5)$. ${ }^{*} \mathrm{P}<0.05$ and ${ }^{* *} \mathrm{P}<0.01 .{ }^{\#} \mathrm{P}<0.05$ and ${ }^{\# \#} \mathrm{P}<0.01$. 

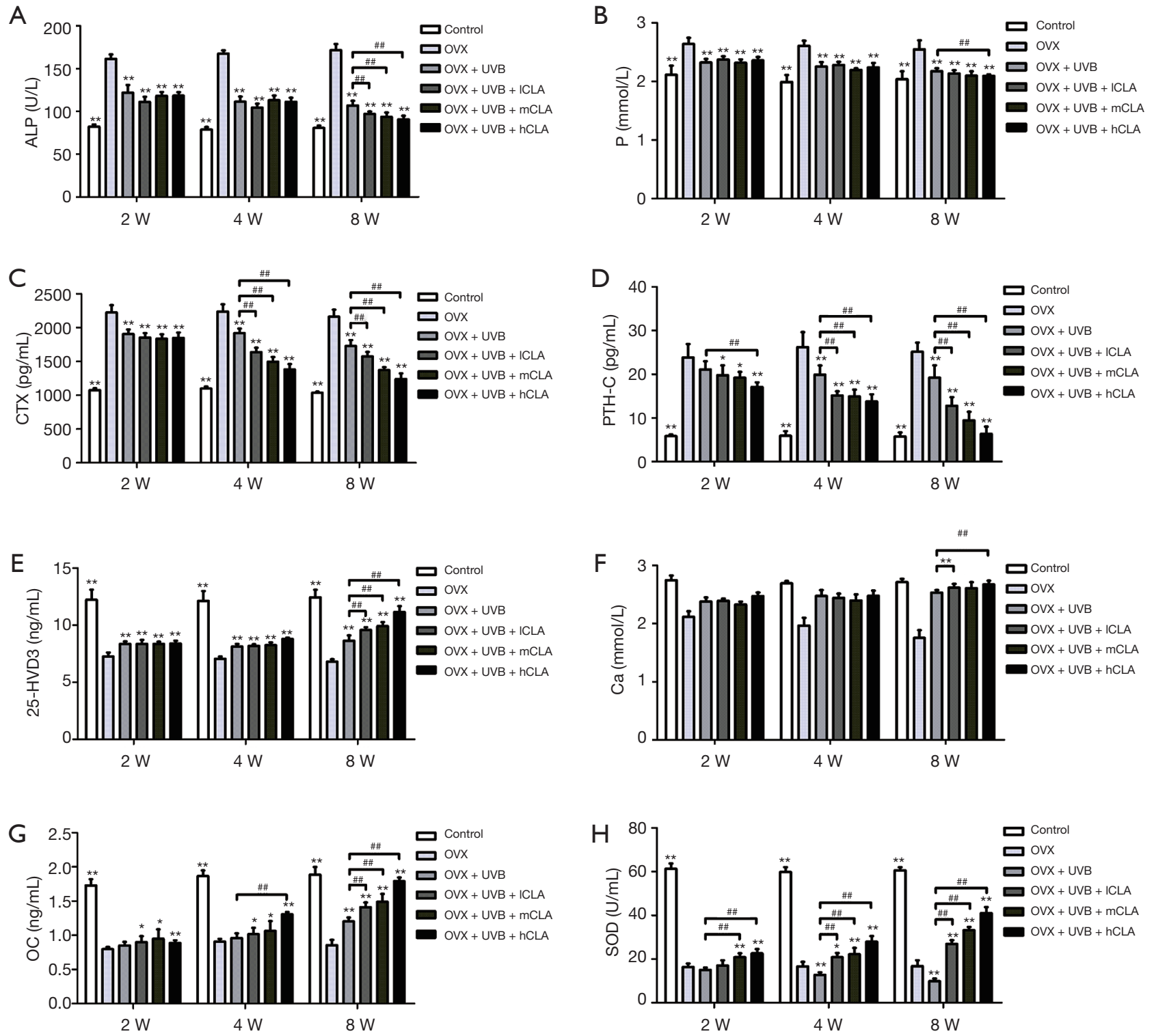

Figure 9 Detection of ALP, phosphorus, CTX, PTH-C, 25-HVD3, calcium, OC, and SOD levels in rat sera. The levels of (A) ALP (n=5), (B) phosphorus (n=5), (C) CTX (n=5), (D) PTH-C (n=5), (E) 25-HVD3 (n=5), (F) calcium (n=5), (G) OC (n=5), and (H) SOD (n=5) in rat sera. Data are shown as the mean \pm standard deviation $(\mathrm{SD}),{ }^{\#} \mathrm{P}<0.05,{ }^{* \#} \mathrm{P}<0.01$. Compared with model group, ${ }^{*} \mathrm{P}<0.05,{ }^{* *} \mathrm{P}<0.01$. ALP, alkaline phosphatase.

related key factors, including 25-HVD3, calcium, and OC, increased (Figure 9). Meanwhile, CLA alleviated UVBinduced oxidative damage and improved SOD concentration (Figure 9). These results indicate that the treatment created an environment suitable for bone regeneration in rat serum, in which more vitamin $\mathrm{D}$ is produced, calcium accumulates, and osteoblasts are strongly activated. These conditions subsequently prompt bone formation and inhibit bone resorption, leading to the eventual relief of osteoporosis. Micro-CT (Figure 7) of rat bone after UVB and CLA treatment also evidences this result. UVB irradiation is cheap and readily available. CLA dietary supplement is also an available treatment method. Moreover, this combination therapy does not entail side effects (gastrointestinal reactions, cancers, or osteonecrosis of the jaw) and reduced skeletal strength, all of which are common with hormone 

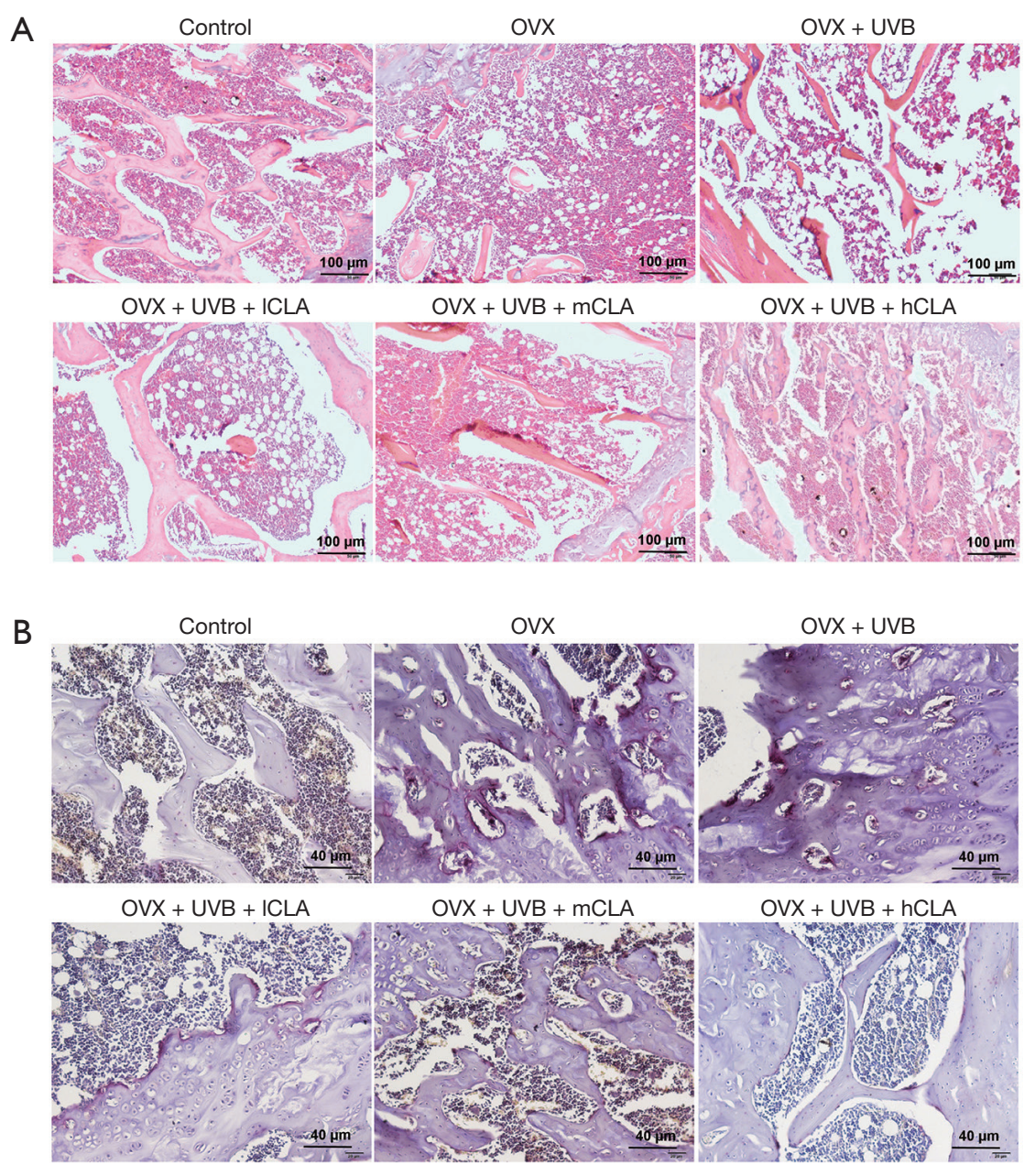

OVX + UVB + hCLA
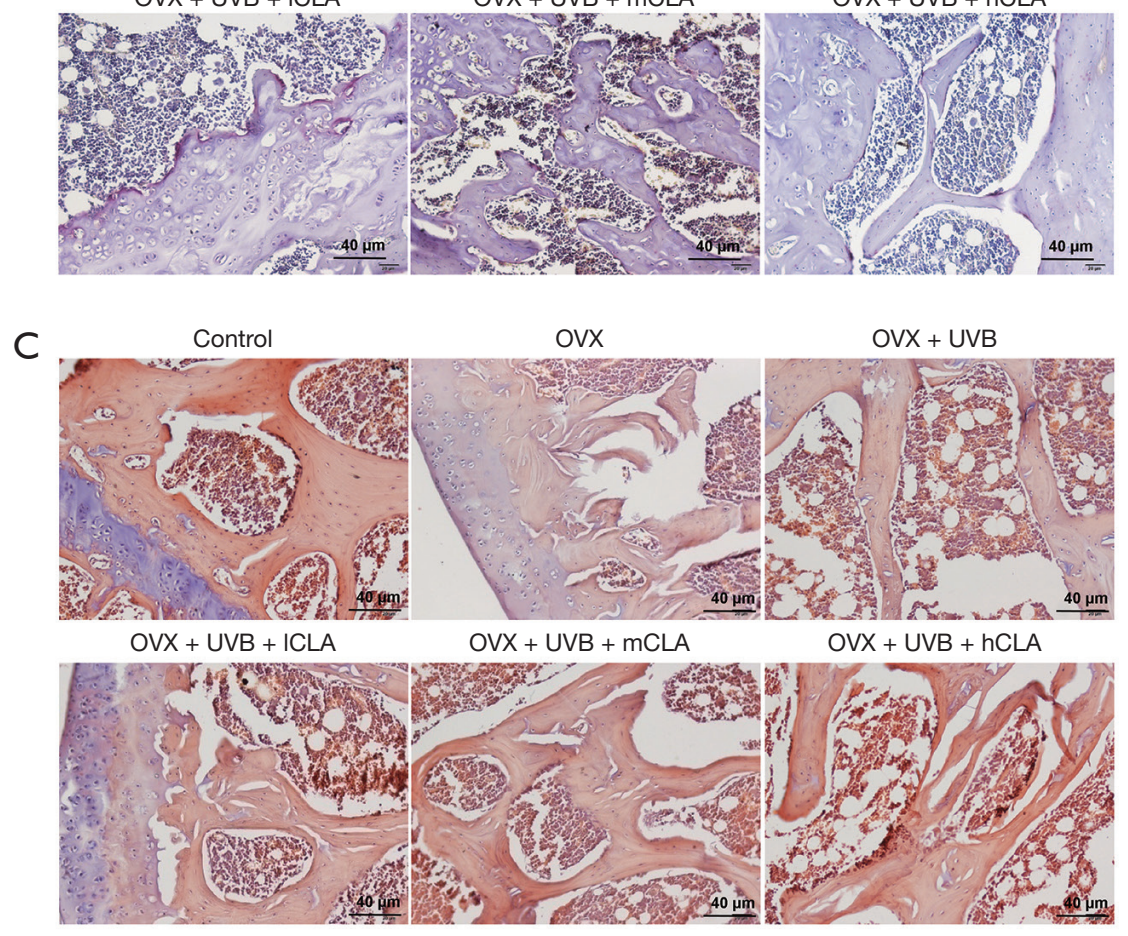

ovx

OVX + UVB

Figure 10 Effects of UVB and CLA on bone density, and TRAP and cellular mineralized nodule expression in rat femurs. (A) Hematoxylineosin staining of bone sections $(n=5)$. Images were captured at $\times 100$ magnification. (B) Immunohistochemical image of TRAP-positive areas of bone sections $(n=5)$. Images were captured at $\times 200$ magnification. (C) Immunohistochemical image of cellular mineralized nodule-positive areas of bone sections $(n=5)$. Images were captured at $\times 200$ magnification. TRAP, tartaric-resistant acidic phosphatase; UVB, ultraviolet B; CLA, conjugated linoleic acid. 
replacement, growth hormone, sodium fluoride, and parathyroid hormone therapy (32). Thus, our work provides a potential alternative treatment regimen for patients with osteoporosis, especially postmenopausal women. Our results also provide a research background to UVB and/or CLAbased osteoporosis therapy.

In this study, we discovered extremely interesting biological interactions between CLA and UV. As evidenced in Figure $9 A, B, C, D$, it was found that UVB irradiation decreased the content of ALP, phosphorus, CTX, and PTH-C in the serum of the OVX + UVB, OVX + UVB + ICLA, OVX + UVB + mCLA, OVX + UVB + hCLA group rats compared with the model groups, whereas CLA strengthened these effects. Although different doses of CLA showed no strong differences after 2 weeks or 4 weeks of treatment, obvious CLA dose-based differences in these effects were observed, which suggested that the treatment effects were time and dose dependent. Also, CLA intensified UVB-induced 25-HVD3, calcium, and osteocalcin production in a dose- and time-dependent manner. It was synergistic. Meanwhile, CLA alleviated UVB-induced oxidative damage (Figure 9H). In Figure 3, ALP serum concentration, ALP staining, cellular mineralized nodule staining, and fluorescence detection of ROS revealed that CLA treatment relieved UVB-triggered ALP inhibition, cellular mineralized nodule reduction, and ROS elevation, thus weakening osteogenic inhibition and oxidative damage. In Figure $6 A, B$, it can be seen that CLA treatment mitigated the TRAP production and promotion of osteoclastic differentiation resulting from UVB irradiation, which significantly inhibited osteoclast formation. Interestingly, the results of ELISA (Figure 6C) showed that CLA effectively inhibited BMMC expression of inflammatory factors (IL-6 and TNF- $\alpha$ ) and promoted the production of anti-inflammatory factor IL-10, inhibiting both the formation of osteoclasts and inflammatory damage to BMMCs induced by UVB $(72 \mathrm{~cm})$ irradiation. It has been reported that UV irradiation causes oxidative damage and elevates the level of ROS to induce apoptotic biochemical alterations (33).

Treatment with UV radiation led to an increase of serum $25(\mathrm{OH}) \mathrm{D}$ concentration, which is the best indicator of the overall vitamin D status of an individual (34). Vitamin D is vital for bone metabolism; thus, vitamin D deficiency contributes to the pathogenesis of osteoporosis and hip fractures (35). However, exposure to UVB can also have side effects on the skin, such as erythema, vasodilation, edema, and angiogenesis (36), and T-cell depletion (37), as well as on the eyes, being a risk factor for cataractogenesis (38). UVB radiation-induced apoptosis has been extensively studied in human keratinocytes, which represent the major cellular target for solar UVB radiation (31). UVB radiation acts as a strong apoptotic trigger for many tumor and normal cells (39). Generation of ROS has been demonstrated to be the major contributing factor to UVBinduced cell death $(18,19)$. The resulting oxidative stress sees the impairment of cellular antioxidants, the induction of DNA damage, and the occurrence of apoptosis. Skin cells undergo apoptosis due to irreversible DNA damage (40), and which can prevent the accumulation of abnormal cells, leading to cutaneous malignancies (41). In this study, we found that UVB caused oxidative damage to BMSCs (Figure 2D) and BMMCs. In vivo study revealed that UVB enhanced oxidative damage by decreasing the level of serum SOD (Figure 9H). In contrast, CLA weakened UVB-caused oxidative damage by decreasing the ROS level (Figure 3D), while increasing the serum SOD level (Figure $9 H)$, inflammatory reaction by decreasing the levels of IL-6 and TNF- $\alpha$, and increasing IL-10 level (Figure 6C). These results strengthened the evidence of the effects of the combination treatment of UVB and CLA on osteoporosis and pave the way for further research on the underlying mechanism.

Over the last several years, considerable research attention has focused on bioactive natural products, including polysaccharides, fatty acids, oleic acids, and linoleic acids, and their biological and immunological activities $(42,43)$. CLA is well known for its capacity to reduce fat masses and body weights (44). In this study, we found that after ovariectomy, the intakes of rats increased significantly and their body weights increased rapidly (Figure $8 B$ and $C$ ), whereas CLA-treated rats showed slow increases in body weight and had decreased intakes. Furthermore, 8 weeks later after CLA treatment the body weight and intakes of OVX osteoporosis rats were close to those of the control group. Many other research groups have demonstrated such a body weight-control effect of CLA $(45,46)$. However, short-term CLA supplementation may not affect body weight or show obvious benefits with respect to bone formation. For instance, Doyle et al. reported that markers of calcium or bone metabolism were not affected by short-term CLA supplementation (27), and Kelly et al. reported that short-term CLA supplementation of an omega-3 PUFA-rich diet enhanced calcium absorption in young growing rats, although it had no assessable effect on bone metabolism or bone mass (47). 


\section{Acknowledgments}

Funding: This work was supported by the Natural Science Foundation of China (grant No. 81760396), the Natural Science Foundation of Qinghai Province (grant No. 2018ZJ-715), and the Science and Technology Foundation Platform of Qinghai Province (grant No. 2020-ZJ-T08).

\section{Footnote}

Reporting Checklist: The authors have completed the ARRIVE reporting checklist. Available at http://dx.doi. org/10.21037/atm-21-934

Data Sharing Statement: Available at http://dx.doi. org/10.21037/atm-21-934

Conflicts of Interest: All authors have completed the ICMJE uniform disclosure form (available at http://dx.doi. org/10.21037/atm-21-934). All authors report funding from the Natural Science Foundation of China (grant No. 81760396), the Natural Science Foundation of Qinghai Province (grant No. 2018-ZJ-715), and the Science and Technology Foundation Platform of Qinghai Province (grant No. 2020-ZJ-T08). The authors have no other conflicts of interest to declare.

Ethical Statement: The authors are accountable for all aspects of the work in ensuring that questions related to the accuracy or integrity of any part of the work are appropriately investigated and resolved. Experiments were performed under a project license (No.: 2018-52) granted by the Ethics Committee of the First Affiliated Hospital of Soochow University, in compliance with the institutional guidelines for the care and use of animals.

Open Access Statement: This is an Open Access article distributed in accordance with the Creative Commons Attribution-NonCommercial-NoDerivs 4.0 International License (CC BY-NC-ND 4.0), which permits the noncommercial replication and distribution of the article with the strict proviso that no changes or edits are made and the original work is properly cited (including links to both the formal publication through the relevant DOI and the license). See: https://creativecommons.org/licenses/by-nc-nd/4.0/.

\section{References}

1. Watts NB. Postmenopausal Osteoporosis: A Clinical
Review. J Womens Health (Larchmt) 2018;27:1093-6.

2. Eastell R, Black DM, Boonen S, et al. Effect of once-yearly zoledronic acid five milligrams on fracture risk and change in femoral neck bone mineral density. J Clin Endocrinol Metab 2009;94:3215-25.

3. Lopez Gavilanez E, Johansson H, McCloskey E, et al. Assessing the risk of osteoporotic fractures: the Ecuadorian FRAX model. Arch Osteoporos 2019;14:93.

4. Wakatsuki A, Ikenoue N, Sagara Y. Effects of estrogen on susceptibility to oxidation of low-density and highdensity lipoprotein in postmenopausal women. Maturitas 1998;28:229-34.

5. Gambacciani M, Cagnacci A, Lello S. Hormone replacement therapy and prevention of chronic conditions. Climacteric 2019;22:303-6.

6. Anastasilakis AD, Polyzos SA, Makras P. Therapy of endocrine disease: Denosumab vs bisphosphonates for the treatment of postmenopausal osteoporosis. Eur J Endocrinol 2018;179:R31-45.

7. Castelo-Branco C, Figueras F, Sanjuan A, et al. Longterm postmenopausal hormone replacement therapy effects on bone mass: differences between surgical and spontaneous patients. Eur J Obstet Gynecol Reprod Biol 1999;83:207-11.

8. Teede HJ, Vincent A. Hormone therapy -- where are we now? Aust Fam Physician 2011;40:280-5.

9. Holick MF. Vitamin D: importance in the prevention of cancers, type 1 diabetes, heart disease, and osteoporosis. Am J Clin Nutr 2004;79:362-71.

10. Erkal MZ, Wilde J, Bilgin Y, et al. High prevalence of vitamin $\mathrm{D}$ deficiency, secondary hyperparathyroidism and generalized bone pain in Turkish immigrants in Germany: identification of risk factors. Osteoporos Int 2006;17:1133-40.

11. Heyburn PJ, Peacock M, Casson IF, et al. Vitamin $\mathrm{D}$ metabolites in post-menopausal women and their relationship to the myopathic electromyogram. Eur J Clin Invest 1983;13:41-4.

12. Trummer C, Pandis M, Verheyen N, et al. Beneficial Effects of UV-Radiation: Vitamin D and beyond. Int J Environ Res Public Health 2016;13:1028.

13. Bacon CJ, Gamble GD, Horne AM, et al. High-dose oral vitamin D3 supplementation in the elderly. Osteoporos Int 2009;20:1407-15.

14. Kragballe K. Vitamin D and UVB radiation therapy. Cutis 2002;70:9-12.

15. Chandra P, Wolfenden LL, Ziegler TR, et al. Treatment of vitamin D deficiency with UV light in patients with 
malabsorption syndromes: a case series. Photodermatol Photoimmunol Photomed 2007;23:179-85.

16. Holick MF, Chen TC, Lu Z, et al. Vitamin D and skin physiology: a D-lightful story. J Bone Miner Res 2007;22 Suppl 2:V28-33.

17. Ferguson G, Gehrmann W. Restoring vitamin D in monitor lizards: exploring the efficacy of dietary and UVB sources. Journal of Herpetological Medicine \& Surgery 2009;19:81-8.

18. Wölfle U, Esser PR, Simon-Haarhaus B, et al. UVBinduced DNA damage, generation of reactive oxygen species, and inflammation are effectively attenuated by the flavonoid luteolin in vitro and in vivo. Free Radic Biol Med 2011;50:1081-93.

19. Halliday GM. Common links among the pathways leading to UV-induced immunosuppression. J Invest Dermatol 2010;130:1209-12.

20. Ma DW, Wierzbicki AA, Field CJ, et al. Conjugated linoleic acid in canadian dairy and beef products. J Agric Food Chem 1999;47:1956-60.

21. Lucatto JN, Brandão SNTGD, Drunkler DA. Conjugated linoleic acid: chemical structure, occurrence and effects on human health. Revista Do Instituto De Latícinios Cândido Tostes 2014;69.

22. Zhang H, Guo Y, Yuan J. Conjugated linoleic acid enhanced the immune function in broiler chicks. Br J Nutr 2005;94:746-52.

23. Kim JH, Kim Y, Kim YJ, et al. Conjugated Linoleic Acid: Potential Health Benefits as a Functional Food Ingredient. Annu Rev Food Sci Technol 2016;7:221-44.

24. Brownbill RA, Petrosian M, Ilich JZ. Association between dietary conjugated linoleic acid and bone mineral density in postmenopausal women. J Am Coll Nutr 2005;24:177-81.

25. Kennedy OD, Herman BC, Laudier DM, et al. Activation of resorption in fatigue-loaded bone involves both apoptosis and active pro-osteoclastogenic signaling by distinct osteocyte populations. Bone 2012;50:1115-22.

26. Rahman MM, Fernandes G, Williams P. Conjugated linoleic Acid prevents ovariectomy-induced bone loss in mice by modulating both osteoclastogenesis and osteoblastogenesis. Lipids 2014;49:211-24.

27. Doyle L, Jewell C, Mullen A, et al. Effect of dietary supplementation with conjugated linoleic acid on markers of calcium and bone metabolism in healthy adult men. Eur J Clin Nutr 2005;59:432-40.

28. Platt I, Rao LG, El-Sohemy A. Isomer-specific effects of conjugated linoleic acid on mineralized bone nodule formation from human osteoblast-like cells. Experimental Biology \& Medicine 2007;232:246-52.

29. Platt ID, El-Sohemy A. Regulation of osteoblast and adipocyte differentiation from human mesenchymal stem cells by conjugated linoleic acid. J Nutr Biochem 2009;20:956-64.

30. Arab K, Rossary A, Soulère L, et al. Conjugated linoleic acid, unlike other unsaturated fatty acids, strongly induces glutathione synthesis without any lipoperoxidation. Br J Nutr 2006;96:811-9.

31. Narayanan DL, Rao NS, Fox JL. Review: Ultraviolet radiation and skin cancer. International Journal of Dermatology 2010;49:978-86.

32. Rozenberg S, Vasquez JB, Vandromme J, et al. Educating patients about the benefits and drawbacks of hormone replacement therapy. Drugs Aging 1998;13:33-41.

33. Chan WH, Wu CC, Yu JS. Curcumin inhibits UV irradiation-induced oxidative stress and apoptotic biochemical changes in human epidermoid carcinoma A431 cells. J Cell Biochem 2003;90:327-38.

34. Karppinen T, Ala-Houhala M, Ylianttila L, et al. The effect of vernal solar UV radiation on serum 25-hydroxyvitamin D concentration depends on the baseline level: observations from a high latitude in Finland. Int J Circumpolar Health 2017;76:1272790.

35. Glowacki J, Hurwitz S, Thornhill TS, et al. Osteoporosis and vitamin-D deficiency among postmenopausal women with osteoarthritis undergoing total hip arthroplasty. J Bone Joint Surg Am 2003;85:2371-7.

36. Hirakawa S, Fujii S, Kajiya K, et al. Vascular endothelial growth factor promotes sensitivity to ultraviolet B-induced cutaneous photodamage. Blood 2005;105:2392-9.

37. Ozawa M, Ferenczi K, Kikuchi T, et al. 312-nanometer Ultraviolet B Light (Narrow-Band UVB) Induces Apoptosis of T Cells within Psoriatic Lesions. J Exp Med 1999;189:711-8.

38. Yoshitomi Y, Osada H, Satake H, et al. Ultraviolet B-induced Otx2 expression in lens epithelial cells promotes epithelial-mesenchymal transition. Biology Open 2019;8:bio035691.

39. Salucci S, Burattini S, Battistelli M, et al. Ultraviolet B (UVB) Irradiation-Induced Apoptosis in Various Cell Lineages in Vitro. Int J Mol Sci 2012;14:532-46.

40. Jackson S, Harwood C, Thomas M, et al. Role of Bak in $\mathrm{UV}$-induced apoptosis in skin cancer and abrogation by HPV E6 proteins. Genes Dev 2000;14:3065-73.

41. Katiyar SK, Mantena SK, Meeran SM. Silymarin protects epidermal keratinocytes from ultraviolet radiation-induced 
apoptosis and DNA damage by nucleotide excision repair mechanism. PLoS One 2011;6:e21410.

42. Roberts DCK. Dietary trans fatty acids and cancer: a review of the evidence. Food Australia Official Journal of Cafta \& Aifst 1995.

43. Jin Y, Li P, Wang F. $\beta$-glucans as potential immunoadjuvants: A review on the adjuvanticity, structureactivity relationship and receptor recognition properties. Vaccine 2018;36:5235-44.

44. Gaullier JM, Halse J, Høye K, et al. Supplementation with conjugated linoleic acid for 24 months is well tolerated by and reduces body fat mass in healthy, overweight humans. J Nutr 2005;135:778-84.

Cite this article as: Shan Z, Zhao Y, Qiu Z, Angxiu S, Gu Y, Luo J, Bi H, Luo W, Xiong R, Ma S, He Z, Chen L. Conjugated linoleic acid prompts bone formation in ovariectomized osteoporotic rats and weakens osteoclast formation after treatment with ultraviolet B. Ann Transl Med 2021;9(6):503. doi: 10.21037/atm-21-934
45. Lee J, Li Y, Li C, et al. Natural products and body weight control. N Am J Med Sci 2011;3:13-9.

46. Blankson H, Stakkestad JA, Fagertun H, et al. Conjugated linoleic acid reduces body fat mass in overweight and obese humans. J Nutr 2000;130:2943-8.

47. Kelly O, Cusack S, Jewell C, et al. The effect of polyunsaturated fatty acids, including conjugated linoleic acid, on calcium absorption and bone metabolism and composition in young growing rats. Br $\mathrm{J}$ Nutr 2003; $90: 743-50$.

(English Language Editor: J. Reynolds) 